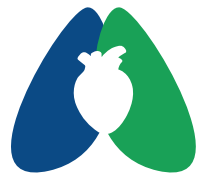

ASSOBRAFIR

CI Ê N C I A

\title{
Correlação entre atividades de vida diária e capacidade funcional de membros superiores e inferiores em pacientes com DPOCa
}

\author{
Correlation between activities of daily life and functional capacity \\ of upper and lower limbs in patients with COPD
}

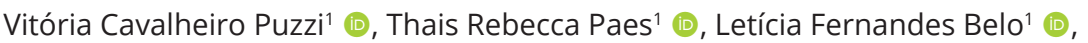

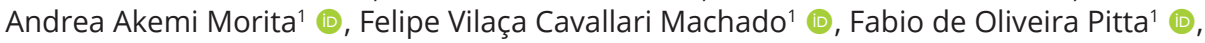

Nidia Aparecida Hernandes ${ }^{1}$ (i)

aApresentação dos dados em evento: Esse estudo foi submetido ao Congresso: IX Congresso Sulbrasileiro de Fisioterapia Respiratória, Cardiovascular e em Terapia Intensiva.

${ }^{1}$ Laboratório de Pesquisa em Fisioterapia Pulmonar (LFIP), Departamento de Fisioterapia, Universidade

Estadual de Londrina (UEL), Londrina, PR, Brasil.

Como citar: Puzzi V, Paes TR, Belo LF, Morita AA, Machado FVC, Pitta FO, et al. Correlação entre atividades de vida diária e capacidade funcional de membros superiores e inferiores em pacientes com dpoc. ASSOBRAFIR Ciênc. 2021;12:e41798. https://doi.org/10.47066/2177-9333.AC.2020.0023

\section{Resumo}

Introdução: Alterações desencadeadas pela Doença Pulmonar Obstrutiva Crônica (DPOC) podem causar sensação de fadiga de membros superiores e inferiores. Entretanto, a maioria dos testes de capacidade funcional envolvem somente os membros inferiores (MMII). Sendo assim, é importante saber se o desempenho na realização das AVDs é similar durante a avaliação de capacidade funcional de MMSS e de MMII. Objetivo: Verificar a correlação entre o desempenho nas AVDs e a capacidade funcional de MMSS e de MMII em pacientes com DPOC. Métodos: Pacientes com DPOC foram avaliados quanto: função pulmonar (espirometria), AVDs (Londrina ADL Protocol-LAP), capacidade funcional de MMII (teste da caminhada de 6 minutos -TC6min) e capacidade funcional de MMSS (teste das argolas de 6 minutos -TA6min) Resultados: Foram incluídos 39 pacientes (20 homens; 64[61-72] anos; IMC: 27,7[23-32] Kg/m²; VEF correlacionou com o TC6min (valores absolutos e em porcentagem da distância percorrida predita). Porém, se correlacionou moderadamente com o número de argolas no TA6min ( $r=-0,46$ P=0,002). A porcentagem do tempo predito do LAP apresentou correlação moderada com a porcentagem do predito do TC6min, com a distância percorrida no TC6min e com o número de argolas no TA6min $(r=0,61 P<0,0001 ; r=0,43 P=0,005 ; r=0,44 P=0,004)$ respectivamente. Conclusão: O desempenho nas AVDs, correlacionou-se moderadamente com a capacidade funcional de MMSS e MMII. Acredita-se que isto se deva ao fato de o protocolo envolver atividades que utilizam as atividades do corpo todo, logo, importantes no cotidiano de pacientes com DPOC.

Palavras-chave: Doença Pulmonar Obstrutiva Crônica; Atividades Cotidianas; Exercício.

\begin{abstract}
Background: Changes caused by chronic obstructive pulmonary disease (COPD) can lead to upper (UL) and lower limb (LL) fatigue, reported by patients during activities of daily living (ADLs). However, most functionality tests involve only LL. Therefore, it is important to know whether the performance in performing ADLs is similar when assessing the functional capacity of UL and LL. Aim: To verify the correlation between performance in ADLs and functional capacity of UL and LL in patients with COPD. Methods: COPD patients underwent evaluation of: pulmonary function (spirometry), ADLs (Londrina ADL Protocol-LAP), functional capacity of LL (6-minute walk test-6MWT) and the functional capacity of UL (6-minute ring test-6MPRT). Results: 39 patients were included (20 men; 64[61-72] years; BMI: 27.7[23-32] Kg/m; $; \mathrm{FEV}_{1}$ : 50[38-57]\% pred). The LAP did not correlate with the 6MWT (absolute values and as a percentage of the predicted distance covered). However, it was moderately correlated with the number of rings in 6MPRT ( $r=-0.46$ $P=0.002$ ). The percentage of the predicted of the LAP presented a moderate correlation with the percentage of the predicted of the 6MWT, with the distance covered in the 6MWT and with the number of rings in the 6MWT $(r=0.61 P<0.0001 ; r=0.43 P=0.005 ; r=0.44 P=0.004)$ respectively. Conclusion: The performance in the ADLs was moderately correlated with the functional capacity of UL and LL. It is believed that this is due to the fact that the protocol involves activities that use the activities of the whole body, therefore, important in the daily lives of patients with COPD.
\end{abstract}

Submissão em: Outubro 01, 2020. Aceito em: Julho 05, 2021

realizado em: Universidade Estadual de Londrina, Londrina, PR, Brasil. Aprovação ética: CAEE 57961716.2.0000.5231 da Universidade Estadual de Londrina, $\mathrm{n}^{\circ} 1.730 .247$.

*Autor correspondente: Nidia Aparecida Hernandes. E-mail: nyhernandes@gmail.com
Keywords: Chronic Obstructive Pulmonary Disease; Daily Activities; Exercise. 


\section{INTRODUÇÃO}

A doença pulmonar obstrutiva crônica (DPOC) é uma doença com um caráter progressivo, porém tratável, caracterizada por uma limitação persistente ao fluxo aéreo, anormalidades nutricionais e disfunção musculoesquelética ${ }^{1}$, como alterações na composição corporal e fraqueza muscular ${ }^{2}$. Esse quadro clínico favorece a sintomatologia comumente referida pelos pacientes durante as atividades cotidianas, como dispneia e sensação de fadiga de membros superiores e inferiores. Tais sintomas comumente induzem à limitação funcional nesses pacientes ${ }^{2}$.

Sabe-se que a disfunção muscular periférica e os sintomas de dispneia e fadiga são fatores importantes que estão relacionados à performance das $\mathrm{AVD} \mathrm{s}^{3}$, comumente relatados pelos pacientes com DPOC. Entretanto, ainda não se sabe se essas atividades são relacionadas com a capacidade funcional de membros superiores (MMSS) ou de membros inferiores (MMII), uma vez que a maioria dos testes que avaliam capacidade funcional, utilizam testes em que os MMII são mais relacionados.

Por outro lado, já existem estudos que mostram que as AVDs que envolvem a utilização dos MMSS (lavar louças, estender roupas) desencadeiam mais sintomas de dispneia para os indivíduos com DPOC 4 . Isso pode ser explicado pela ação dos músculos acessórios da respiração que são recrutados nos movimentos de MMSS, e que exercem também a função de estabilizar a caixa torácica e tronco ${ }^{5}$. Sendo assim, é importante saber se o desempenho na realização das AVDs está relacionado com a capacidade funcional de MMSS e de MMII afim de direcionar as condutas fisioterapêuticas profiláticas e/ou reabilitadoras, para melhor manejo da doença. Sabendose dessa necessidade, o objetivo do estudo foi verificar a correlação entre o desempenho nas AVDs e a capacidade funcional de MMSS e de MMII em pacientes com DPOC.

\section{MÉTODOS}

\section{Amostra}

Neste estudo transversal, foram incluídos pacientes com DPOC que foram recrutados por meio de uma amostragem de conveniência, nos ambulatórios de Pneumologia e de Fisioterapia Respiratória dos hospitais das Clínicas e Universitário da UEL, respectivamente. Todos os indivíduos incluídos no estudo assinaram um termo de consentimento livre e esclarecido, sendo este o mesmo de um estudo longitudinal ao qual a presente subanálise está atrelada. O estudo longitudinal foi aprovado pelo Comitê de Ética em Pesquisa da Universidade Estadual de Londrina sob o parecer CAEE 57961716.2.0000.5231 da Universidade Estadual de Londrina, $\mathrm{n}^{\circ}$ 1.730.247.

Os critérios de inclusão foram: diagnóstico clínico de DPOC, estabelecido conforme os critérios da Global
Initiative for Chronic Obstructive Lung Disease (GOLD) ${ }^{6}$; estabilidade clínica, sem infecções e exacerbações no último mês; ausência de doença cardíaca grave e/ou instável; ausência de alterações ósteo-neuro-musculares que pudessem limitar as AVDs e atividades físicas de vida diária; e não ter seguido nenhum tipo de programa de exercícios físicos no último ano. Os critérios de exclusão foram: não realização dos testes propostos por qualquer motivo; manifestação do desejo de deixar o estudo em algum momento por qualquer razão.

Os pacientes realizaram dois dias de avaliações, sendo no primeiro realizado o exame de função pulmonar por meio da espirometria e avaliação das AVDs utilizandose o LAP. Já no segundo dia, foram aplicados os testes de capacidade funcional de MMSS e de MMII (teste de caminha de 6 minutos e teste das argolas de 6 minutos). Todos os testes estão descritos a seguir.

\section{Avaliações}

\section{Função Pulmonar}

A avaliação da função pulmonar foi realizada utilizandose um espirômetro (Spirobank G ${ }^{\circledR}$, MIR, Itália), foi realizada de acordo com as diretrizes da American Thoracic Society? Foram utilizados os valores de referência de Pereira et $a l$.

\section{Atividade da Vida Diária}

Para avaliação objetiva das AVDs foi aplicado o Londrina $A D L$ Protocol (LAP) (Figura 1) que é composto por cinco atividades que são realizadas em forma de circuito, sendo que o tempo de execução do protocolo foi registrado por um cronômetro. Este protocolo foi criado pelo presente grupo de pesquisa e já foi validado para pacientes com DPOC $^{8}$.

Para a realização do LAP, o paciente inicia o circuito de atividades em pé no ponto inicial, caminha três metros até uma mesa com objetos, senta-se em uma cadeira sem apoio para MMSS e transfere 10 objetos (peso varia de $250 \mathrm{~g}$ a $2 \mathrm{~kg}$ ) de um lado da mesa para o outro e depois retorna os mesmos objetos para o ponto inicial. Em seguida, caminha 1,5 metros até o ponto onde estão duas sacolas que, juntas, contém $10 \%$ do peso corporal do indivíduo dividido igualmente entre ambas as sacolas; o indivíduo pega então as sacolas e caminha em um corredor de seis metros três vezes, deixando as sacolas ao final da caminhada. Então, caminha 3,5 metros até uma estante e transfere 12 objetos (peso varia de $250 \mathrm{~g}$ a $2 \mathrm{Kg}$ ) que estão em uma mesa para estante e depois retorna os mesmos objetos para a mesa. Para executar a próxima atividade, o indivíduo caminha quatro metros até um varal e estende 10 peças de roupa que estão em um cesto no chão e depois recolhe as peças de roupa, retornando-as para o cesto. Finalmente, caminha 1,5 metros até um corredor de seis metros onde caminha livre de pesos por três vezes, conforme mostrado na Figura 1. Enquanto 


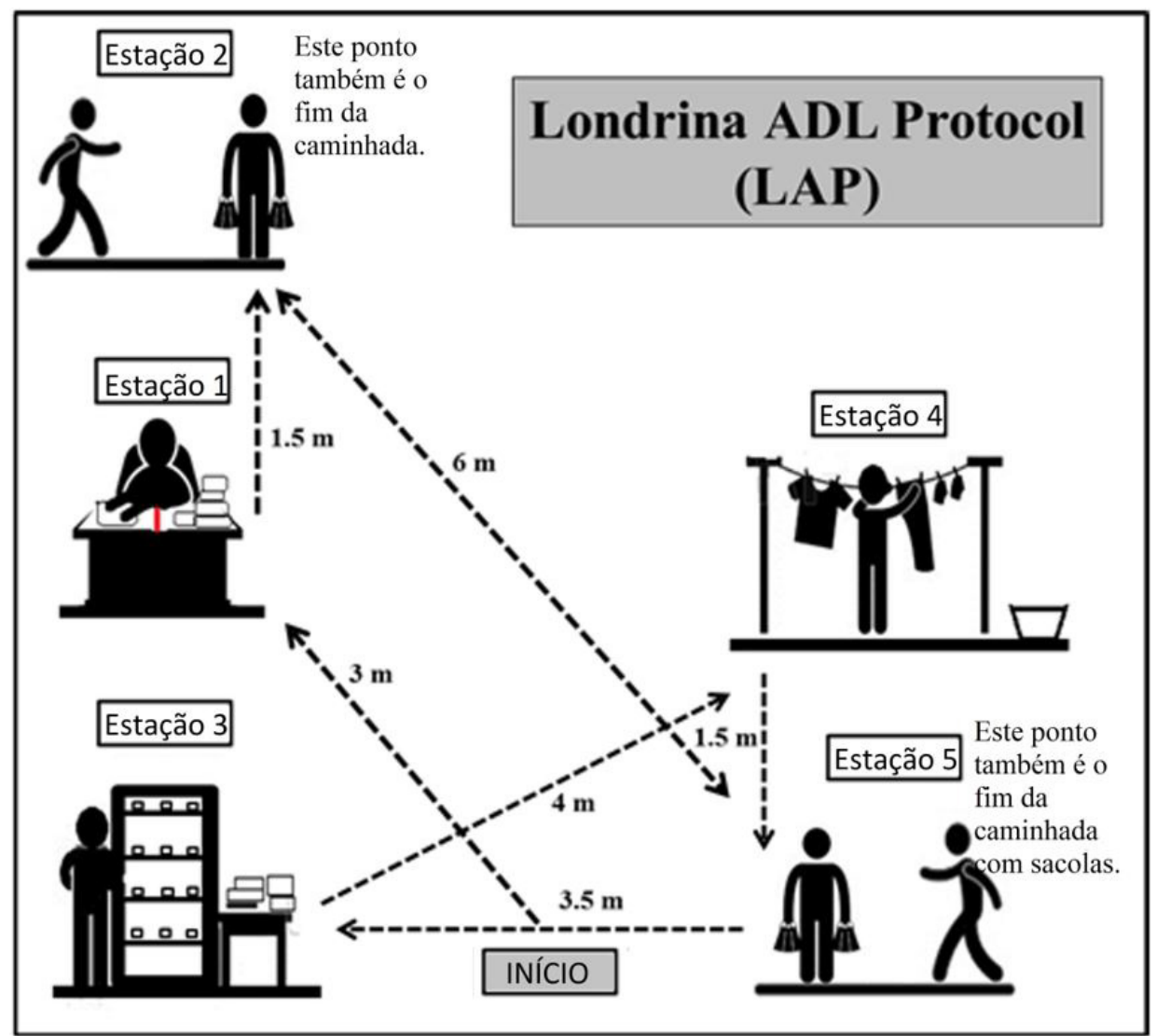

Figura 1. Circuito do protocolo LAP.

o paciente realizava o LAP, o tempo de execução foi registrado pelo avaliador. Todas as atividades foram previamente explicadas e demonstradas pelo avaliador a cada participante.

O tempo gasto pelo indivíduo para concluir o circuito foi utilizado para as análises. Além disso, frequência cardíaca, pressão arterial e sensação de dispneia e fadiga (escala de Borg modificada para esforço percebido ${ }^{6}$ ) foram avaliados antes e após a execução do LAP.

\section{Capacidade funcional de MMII e MMSS}

O teste de caminhada de 6 minutos foi utilizado para avaliar a capacidade funcional de MMII, de acordo com padronizações internacionais. Os indivíduos foram orientados a caminhar e percorrer a maior distância possível em 6 minutos em um corredor plano de 30 metros de extensão. Foram realizados dois testes com intervalo mínimo de 30 minutos entre eles, sendo o teste com maior distância percorrida utilizado para as análises. Os valores de referência utilizados foram os de Britto et al. ${ }^{9}$.

O teste das argolas de 6 minutos foi utilizado para avaliar a capacidade funcional de MMSS, seguindo a padronização proposta por Zhan et al. ${ }^{10}$. Durante o teste, os indivíduos ficaram sentados de frente para uma placa que contém quatro pinos; dois pinos foram posicionados na altura do ombro do paciente e os outros dois $20 \mathrm{~cm}$ acima. Em cada um dos dois pinos inferiores foram colocadas 10 argolas. Os pacientes foram orientados a mover o número máximo de argolas possível dos dois pinos inferiores para os dois pinos superiores, usando as duas mãos simultaneamente. Quando todas as argolas eram movidas para os pinos da parte superior, os indivíduos deveriam inverter o sentido e trazer as argolas para os pinos inferiores, e assim sucessivamente. A pontuação foi dada pelo número de argolas que o paciente conseguiu mover em um tempo de seis minutos. Frases de encorajamento padronizadas foram ditas ao participante a cada minuto. Foi orientado aos pacientes mover algumas vezes as argolas de baixo para cima e de cima para baixo antes que o teste iniciasse, com o intuito de se familiarizar com o protocolo. Foram realizados 2 testes com um intervalo de 30 minutos e considerado o melhor test para as análises.

\section{Análise estatística}

O teste de Shapiro-Wilk foi utilizado para analisar a distribuição dos dados. Os dados que apresentaram distribuição normal foram descritos em média \pm desviopadrão e os dados que apresentaram distribuição nãonormal, em mediana [intervalo interquartílico 25\%-75\%]. Para verificar correlação entre a capacidade funcional de MMSS e MMII e o tempo de execução do LAP, foi utilizado o coeficiente de correlação de Pearson ou Spearman, de acordo com a distribuição dos dados. O valor de significância estatística adotado foi de $P<0,05$. 


\section{RESULTADOS}

Foram recrutados 42 indivíduos com diagnóstico de DPOC e inclusos 39 nas análises (três foram excluídos por não terem realizado o LAP durante o protocolo do estudo longitudinal), cuja as características estão descritas na Tabela 1. No geral, apresentaram capacidade funcional relativamente preservada e realizaram o LAP mais lentamente do que indivíduos saudáveis de mesma faixa etária, ou seja, tiveram um tempo de execução do protocolo maior do que o previsto (Tabela 1).

O tempo de execução do LAP não apresentou correlação estatisticamente significante com a distância percorrida no TC6 min, em metros ( $r=-0,24 ; P=0,12)$ e em porcentagem do previsto $(r=-0,29 ; P=0,06)$. Já com o número de argolas movidas no TA6min houve estatisticamente significante e moderada ( $r=-0,46 ; P=0,002)$. A porcentagem do previsto do $O$ tempo de execução do LAP, em porcentagem do previsto, correlacionou-se moderadamente com o TC6min, em porcentagem do previsto $(r=0,61 ; P<0,0001)$ e em metros ( $r=0,43 ; P=0,005)$, bem como com o número de argolas no TA6min $(r=0,44 ; P=0,004)$. As correlações podem ser observadas no gráficos da Figura 2 .

\section{DISCUSSÃO}

Neste estudo, foram encontradas correlações moderadas entre desempenho nas AVDs, avaliado pelo LAP, e a capacidade funcional de MMSS e MMII (TA6min e TC6min, respectivamente). O protocolo utilizado para avaliar as AVDs, o LAP, foi recentemente validado e até o momento poucos estudos na literatura foram publicados utilizando essa forma de avaliação. Seu principal diferencial frente aos demais protocolos de AVDs previamente descritos na literatura é a forma de execução das atividades em velocidade usual, ou seja,

Tabela 1. Características dos indivíduos do estudo.

\begin{tabular}{cc}
\hline & $\mathrm{n}=39$ \\
\hline Gênero (H/M) & $20 / 19$ \\
Idade (anos) & $65 \pm 7$ \\
Altura (m) & $159 \pm 0.9$ \\
Peso (kg) & $70 \pm 12$ \\
IMC (kg.m $\left.{ }^{-2}\right)$ & $27,7 \pm 5$ \\
VEF $_{\mathbf{1}} / \mathrm{CVF}$ & $49 \pm 9$ \\
VEF $_{\mathbf{1}}$ (\%prev) & $50 \pm 13$ \\
GOLD (I/II/III/IV) & $0 / 21 / 15 / 3$ \\
TC6min (m) & $467 \pm 80$ \\
TC6min (\%pred) & $88 \pm 15$ \\
TAG6min (número de argolas) & $385 \pm 77$ \\
LAP(segundos) & $321 \pm 64$ \\
LAP(\%prev) & $107 \pm 16$
\end{tabular}

H: homem; M: mulher; IMC: índice de massa corpórea; VEF, volume expiratório forçado no primeiro segundo; CVF: capacidade vital forçada; TC6: teste da caminha de 6 minutos; TA6min: teste de argolas de 6 minutos; LAP: Londrina ADL protocol.
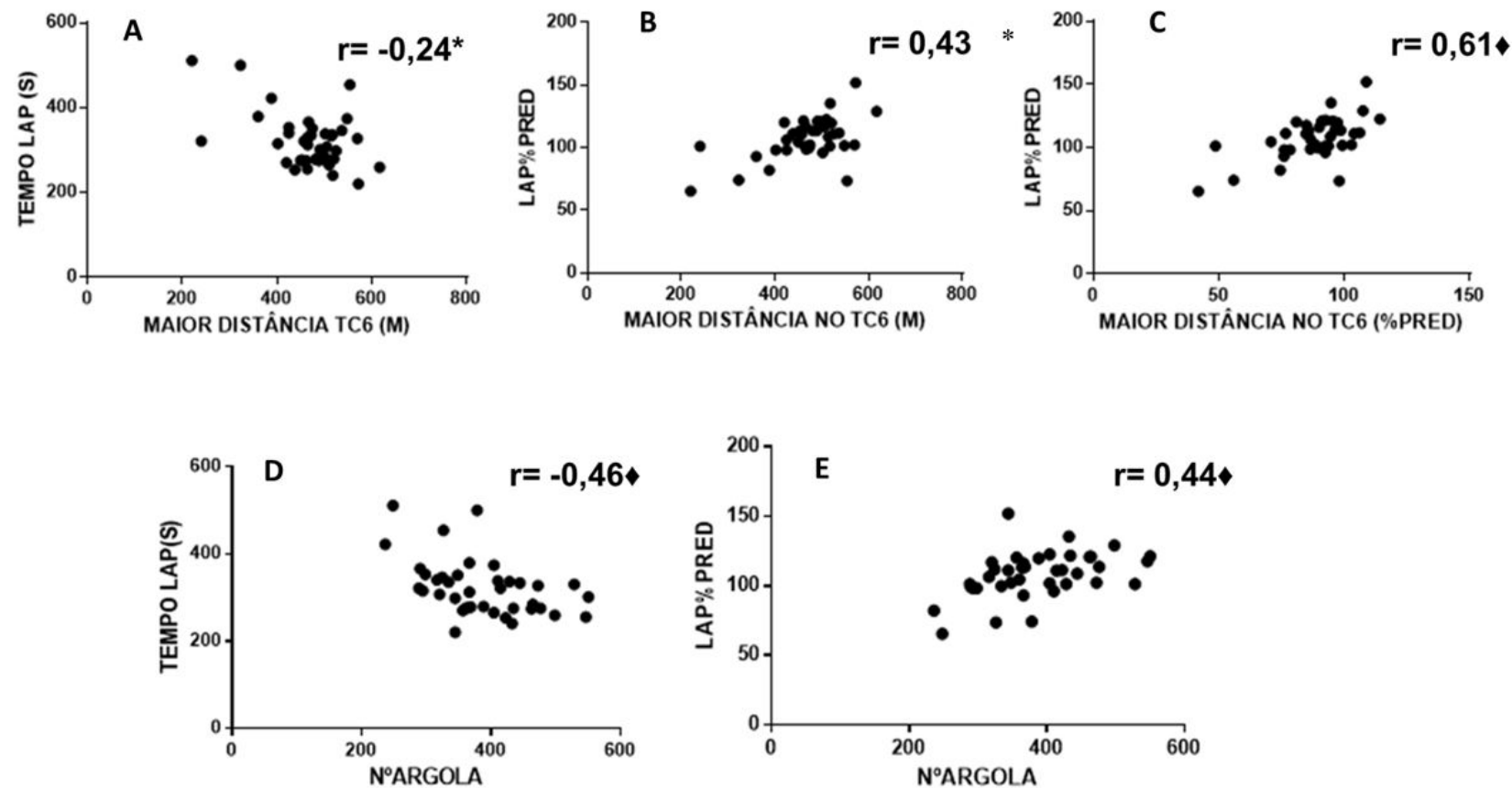

Figura 2. Correlações entre o desempenho nas AVDs e capacidade funcional de MMSS e MMII.

A: Correlação entre TC6min (m) com o tempo em segundos do LAP (seg); B: Correlação entre TC6min (m) com o LAP (\%pred); C: Correlação entra TC6min (\%prev) com o LAP (\%prev); D: Correlação entre TA6min (argolas) com o LAP (segundos); E: Correlação entre TA6min(argolas) com o LAP(\%prev); $\star P=0,12 ; \triangleleft P<0,005$. 
no ritmo em que os pacientes realizam suas tarefas no dia a dia.

Não foi encontrada correlação entre o tempo de execução do LAP, em valores absolutos, e a distância percorrida no TC6min. Resultados diferentes foram observados em dois estudos prévios. Primeiramente, o estudo de Paes et al. ${ }^{11}$ que evidenciou uma correlação moderada entre o tempo de execução do LAP, em segundos, e a distância percorrida no TC6min realizados idosos aparentemente saudáveis. Adicionalmente, no estudo de Sant'Anna et al. ${ }^{8}$, foi evidenciada uma correlação moderada do tempo de execução do LAP, em segundos, com o TC6min realizados por pacientes com DPOC. Apesar de os resultados do presente estudo não corroborarem com os estudos prévios, quando os valores do tempo de execução do LAP foram analisados em porcentagem do valor predito para os indivíduos, correlações moderadas também foram observadas com a distância percorrida no TC6min.

Correlações mais fortes foram encontradas na literatura entre o teste AVD-Glittre e a distância percorrida no TC6min em indivíduos com DPOC ${ }^{12}$. Isso pode ser explicado pelo fato de que nos dois testes a instrução dada ao paciente é que o mesmo deve realizá-lo o mais rápido possível, o que não acontece no LAP. Então, uma possível explicação para o fato de as correlações entre o LAP e os testes de capacidade funcional de MMSS e MMII utilizados no presente estudo terem variado de 0,43 a 0,61 é que o ritmo no qual os indivíduos realizaram os três testes foi diferente. É importante ressaltar que a avaliação do desempenho funcional, este que envolve outros aspectos além da capacidade física do indivíduo ${ }^{13}$, deve refletir de maneira mais abrangente possível o modo pelo qual o indivíduo experencia suas atividades em seu cotidiano, sendo essa uma das justificativas para o LAP ser executado em velocidade usual, segundo seus autores ${ }^{8}$

Foi encontrada uma correlação moderada entre capacidade funcional de MMSS com o protocolo, o que já era esperado, uma vez que a maioria das atividades do protocolo utilizaram os MMSS. No estudo de Takeda et al. ${ }^{14}$, foram encontradas correlações moderadas entre o TA6min e questionários que avaliam as AVDs, assim como o nível de AFVDs. Não foram encontrados estudos que investigaram a correlação entre o TA6min e a capacidade funcional de membros superiores, o que indica a necessidade de mais estudos que investiguem atividades de vida diária e testes de capacidade funcional de MMSS em pacientes com DPOC, uma vez que já foi evidenciado que atividades quem envolvem MMSS desencadeiam maior sensação de dispneia nessa população4 .

Como limitações do presente estudo, pode-se citar a representatividade da amostra, uma vez que os participantes apresentavam capacidade de exercício relativamente preservada (maior que $80 \%$ do predito, em média). Adicionalmente, não havia pacientes com doença leve (GOLD I) na amostra. Sendo assim, pesquisas futuras que incluam pacientes menos e mais graves acrescentariam novas informações ao presente estudo.

Como implicações clínicas do estudo, pode-se trazer a prática clínica a importância do treinamento tanto de MMSS como de MMII em pacientes com DPOC, para que assim estes possam ter um melhor desempenho nos testes de capacidade funcional que predizem uma melhora nas AVDs.

\section{CONCLUSÃO}

O presente estudo evidenciou correlação moderada entre o desempenho nas AVDs, avaliado por meio de um protocolo objetivo (LAP), e a capacidade funcional de MMSS e MMII, indicando que atividades que utilizam tanto a parte superior quanto a inferior do corpo são importantes para o estado funcional de pacientes com DPOC. Sendo assim, chama-se a atenção para a necessidade de se investigar limitações na capacidade funcional de ambos MMSS e MMII durante a avaliação inicial de programas de reabilitação pulmonar a fim de se individualizar a prescrição do treinamento físico.

\section{FONTE DE FINANCIAMENTO}

Nada a declarar.

\section{CONFLITO DE INTERESSES}

Nada a declarar.

\section{AGRADECIMENTOS}

Agradeço aos pesquisadores do LFIP por contribuírem com o meu aprendizado, me auxiliando desde o princípio na elaboração do trabalho e por primarem pela qualidade.

\section{REFERÊNCIAS}

1. Associação Latino-americana de Tórax. Guia de Bolso para o Diagnóstico, a Conduta e a Prevenção da DPOC. Instituto Nacional do Coração, Pulmão e Sangue (NHLBI). Institutos Nacionais de Saúde. Departamento Norte-americano de Serviços Humanos e de Saúde Serviço Público de Saúde. Publicação do NHI No. 2701B.

2. Dourado VZ, Tanni SE, Vale SA, Faganello MM, Sanchez FF, Godoy I. Systemic manifestations in chronic obstructive pulmonary disease. J Bras Pneumol. 2006;32(2):161-71. http://dx.doi.org/10.1590/S1806-37132006000200012. PMid:17273586.

3. Velloso M, do Nascimento NH, Gazzotti MR, Jardim JR. Evaluation of effects of shoulder girdle training on sthength and performance of activities of daily living in patient with chronic obstructive pulmonary disease. Int J Chron Obstruct Pulmon Dis. 2013;8:187-92. PMid:23589685.

4. Souza LM, Gazzotti MR, Sampaio LMM. Relação entre estrutura e função muscular de membros superiores 
em pacientes com doença pulmonar obstrutiva crônica [dissertação]. São Paulo: Universidade 9 de Julho; 2011.

5. Lorenzo VA, Silva AB, Sampaio LM, Jamarni M, Oishi J, Costa D. Efeitos do treinamento físico e muscular respiratório em pacientes com doença pulmonar obstrutiva crônica (DPOC) grave submetidos a BIPAP. Rev Bras Fisioter. 2003;7(1):6976.

6. Global Initiative for Chronic Obstructive Lung Disease [Internet]. GOLD; 2014 [citado em 2014 Jun 10]. Disponível em http://www.goldcopd.org/guidelines-global-strategy-fordiagnosis-management.html

7. Pereira CA, Sato T, Rodrigues SC. New reference values for forced spirometry in white adults in Brazil. J Bras Pneumol. 2007;33(2):397-406. http://dx.doi.org/10.1590/S180637132007000400008. PMid:17982531.

8. Sant'anna T, Donária L, Furlanetto KC, Morakami F, Rodrigues A, Grosskreutz T, et al. Development, Validity and Reliability of the Londrina Activities of Daily Living Protocol, COPD. Respir Care. 2017;62(3):288-97. http://dx.doi.org/10.4187/ respcare.05058. PMid:28143964.

9. Britto RR, Probst VS, de Andrade AF, Samora GA, Hernandes $\mathrm{NA}$, Marinho PE, et al. Reference equations for the sixminute walk distance based on a Brazilian multicenter study. Braz J Phys Ther. 2013;17(3):556-63. http://dx.doi. org/10.1590/S1413-35552012005000122. PMid:24271092.
10. Zhan S, Cerny FJ, Gibbons WJ, Mador MJ, Wu YW. Development of an unsupported arm exercise test in patients with chronic obstructive pulmonary disease. J Cardiopulm Rehabil. 2006;26(3):188-90. http://dx.doi.org/10.1097/00008483200605000-00013. PMid:16738459.

11. Paes TR. Londrina ADL protocol (LAP): reprodutibilidade, validade e valores de referência em adultos fisicamente independentes com 50 anos ou mais [dissertação de mestrado]. Londrina: Universidade Estadual de Londrina; 2016. 68 p.

12. Corrêa KS, Karloh M, Martins LQ, Santos K, Mayer AF. Can the Glittre ADL test differentiate the functional capacity of COPD patients from that of healthy subjects? Rev Bras Fisioter. 2011;15(6):467-73. http://dx.doi.org/10.1590/ S1413-35552011005000034. PMid:22094546.

13. Leidy NK. Functional status and the forward progress of merry-gorounds: toward a coherent analytical framework. Nurs Res. 1994;43(4):196-202. http://dx.doi. org/10.1097/00006199-199407000-00002. PMid:8047422.

14. Takeda K, Kawasaki Y, Yoshida K, Nishida Y, Harada T, Yamaguchi $\mathrm{K}$, et al. The 6-minute pegboard and ring test is correlated with upper extremity activity of daily living in chronic obstructive pulmonary disease. Int J Chron Obstruct Pulmon Dis. 2013;8(2):347-51. http://dx.doi.org/10.2147/ COPD.S45081. PMid:23901268. 